\title{
Research on the Governance Structure of Little Science based on Network of Employment Relationship: Policy Implications for China
}

\author{
Zhen-yu Huang, Yun-long Ding \\ School of Management, Harbin Institute of Technology, 150001, China \\ hzyhit@163.com
}

\begin{abstract}
The composition of the relationships between Scientists and Employers are formal relationship and informal relationship. The asset nature of these two relationships determines the governance structure of the organization. Based on the theory of the transaction cost and institutionalism, Author develops repeated game models and argues that the governance structure of Little Science should be the combination of nonintegration and non-relational contract. Author shows that the governance structure of China's Little Science is improperly built, which should be rebuilt by de-administrative.
\end{abstract}

Keywords: asset nature; de-administration; governance structure; little science; relationship between scientists and employers; repeated game models

\section{Introduction}

With the influence of Prices book Little Science Big Science, modern scholars usually define the term Little Science as a knowledge production model, which characters are free exploring, doing science just for science, the pursuit of truth, doing research by individuals or small groups, etc., while Big Sciences characters are big machine, big team, multidisciplinary, government intervention and so on [1].

Recently, more and more literatures in China believe that Little Science doesnt meet the development demand of modern science, and a full-scale of Big Science management system should be built [2]. This belief, however, has deviated the original intent of Price [3].

Based on the theory of Transaction Cost and Institutionalism, we develop repeatedgame models showing how an ideal governance structure of little science should be. We also show that the improper governance structure of little science in China leads to inefficiency.

\section{Scientist-employer Relationship, Asset Nature and Governance Structure}

In the model of little science, scientist usually research by his own will or interest instead of the orders. For example, if a university employs a professor, what this university actually wants is the fame and status, but not the direct usage of the professor's research findings. The relationship between scientist and employer can be described as organizational relationship, which includes formal relationship and informal relationship.

In transaction cost economics (TCE), asset nature is the key factor of how an organization chooses its formal governance structure. Asset nature means, without sacrificing the producing value, the degree of the asset that can be used for different purposes or used by different people [4]. The higher the degree is, the more special the asset nature will be, while the lower the degree is, the more generality the asset nature will be [5]. Klein, Crawford and Alchian had present further that specialty or generality of 
the asset nature should be viewed from the relationship between the two parties in a transaction [6]. Accordingly, we can find that the asset nature in little is generality for the relationship between scientists and employers.

In institutionalism, however, the function of formal relationship is to help the organization with gaining the legitimacy from a given institutional environment, while informal relationship is the actual mechanism that operates [7]. With this observation, relational contract has been brought out to study relational governance in the literatures of TCE $[8,9,10]$. In these literatures, relational contract is considered to be constituted by mutual benefit, trust, joint action, etc. The empirical studies have proved that asset nature is also the key factor to whether a relational contract will be adopted as the governance structure of informal relationship [11, 12]. In this view, the governance structure of informal relationship between scientists and employers had better adopt non-relational governance.

There are contradictions of the two observations above. On the one hand, the literatures of TCE have studied the function of asset nature in the formal and informal relationships respectively, but it is not clarified that when facing the same nature of one asset, whether two kind of governance structure will be determined at the same time. For instance, in little science, although the nature of the knowledge product is relational generality, it doesn"t mean that non-integration governance and non-relational governance will be coestablished; "non-integration governance + relational governance" or "integration governance + non-relational governance" may also be possible. Because, on the other hand, in the opinion of institutionalism, when facing the same nature of one asset, the decision maker only needs to consider whether adopt relational governance for the informal relationship, While the formal relationship is determined by the legitimacy requirements. To solve the contradictions, we develop repeated game models and establish our main propositions.

\section{Repeated Game Models and Comparative Analysis of Governance Structures}

\subsection{Basic Models}

In the past, scientists were assumed to be selfless and did research for the truth of the world only. Our repeated game models cannot be developed in such assumptions. Because, human are assumed to be bounded rationality and opportunism in TCE, otherwise any organizational design is unnecessary. Luckily, more and more literatures in sociology and economics have released the ideal behavioral assumption of scientists. Now, the scientists are no longer considered to be pure selfless but selfish, and their motivations to do research are diversified and mundane $[13,14]$. Due to these released assumptions, the behavior of the scientists can be viewed as bounded rationality and opportunism, which satisfies the conditions to build our repeated game models for the Scientist-Employer Relationship of little science.

Now, let us consider an environment consisting of a scientist who owns human capital and an employer who uses the product produced by the scientist. The two parties will live forever. The scientist and the employer are risk-neutral. An interest rate $r$ is shared per period. In each period, the scientist will produce some kind of "knowledge product" valued by the employer. As the products" asset nature is relational generality in little science, they are only different in quality (high or low), but not in specified or alternative use. The product"s value will be zero at the end of the period that it belongs to.

For the formal governance structure, we use Williamson"s definitions of nonintegration and vertical integration [15]. Non-integration refers to the independent ownership adopted by scientist and employer, which means the two parties make their own decisions separately. While vertical integration refers to scientist and employer are 
merged into a joint ownership, which means the merger has the ex post right to implement intervention.

As we present before, informal governance structure can be interpreted as relational contract or relational governance that compensates for both parties through trust and mutual benefit. For example, employer can give some kind of bonus to the scientist according to the quality of the product. Thus, given the relational generality product, we need to find out what combinations of the governance structures will be in little science.

Considering the relational combinations in the contradictions above, we need to analyze four forms of governance structures, as shown in Tab. 1:

"G" refers to the asset nature of the product produced by the scientist is Generality. SGN refers to the governance structure consisted of "independent ownership + nonrelational contract". SGI refers to the governance structure consisted of "joint ownership + non-relational contract"( we use "spot" to refer to non-relational contract, because spot can vividly describe the disconnection and independence between scientist and employer). RGN refers to the governance structure consisted of "independent ownership + relational contract". And at last, RGI refers to the governance structure consisted of "joint ownership + relational contract”.

Table 1. Four Forms of Governance Structure

\begin{tabular}{|l|c|c|c|}
\hline & & \multicolumn{2}{|c|}{ Formal Relationship } \\
\hline & & Non-integration (N) & Integration (I) \\
\hline \multirow{2}{*}{ Informal Relationship } & Spot (S) & SGN & SGI \\
\cline { 2 - 4 } & Relationship (R) & RGN & RGI \\
\hline
\end{tabular}

Each period, the Scientist chooses actions $a=\left(a_{1}, a_{2} \ldots a_{n}\right)$ at cost $c(a)$, which will affect the product"s value $Q_{i}(i=H, L)$, in which $Q_{H}$ refers to high value or high quality, $Q_{L}$ refers to low value or low quality, and $Q_{L}<Q_{H}$. It is assumed that the probability of realizing $Q_{H}$ is $q(a)$, then the probability of realizing $Q_{L}$ is $1-q(a)$. We write $\Delta Q=Q_{H^{-}} Q_{L}$, and, $\mathrm{Q}_{\mathrm{H}}=\mathrm{Q}_{\mathrm{L}}+\mathrm{q}(\mathrm{a}) \Delta \mathrm{Q}$. It is further assumed that $c(0)=0$ and $q(0)=0$, which means that when the scientist fails to take any actions, he pays nothing as well as having no chance of getting $Q_{H}$.

When scientist"s first-best actions a* maximize the expected value of the product in its efficient use minus the cost of actions, $Q_{L}+q(a) \Delta Q-c(a)$, and so produce total surplus: $S^{*}=Q_{L}+q\left(a^{*}\right) \Delta Q-c\left(a^{*}\right)$.

As restricted by the bounded rationality, the actions are unobservable to anyone but the scientist himself, so contracts on actions can"t be enforced. We further assume that this contract can"t be enforced by a third party. On the other hand, $Q$ can be observed by the scientist and employer, so that four different forms of governance structure will influence the scientist"s choices of actions.

3.1.1 SGN: In SGN, once the scientist takes actions, he either produces $Q_{H}$ or $Q_{L}$. Although scientist and employer cannot make a complete contract for the realizing of $Q_{H}$ and $Q_{L}$ directly, they can negotiate over the price of the product. Here, Nash bargaining solution is used to arrive at the price: employer will pay scientist the low quality value, $Q_{L}$, plus half of the surplus from use by the employer, $Q_{i}-Q_{L}$, so the price is $Q_{L}+1 / 2 q(a) \Delta Q$.

In SGN, the scientist"s payoff is the price $Q_{L}+1 / 2 q(a) \Delta Q$ less the cost of actions $c(a)$. Scientist therefore chooses actions a ${ }^{\mathrm{SGN}}$ to solve:

$$
U^{S G N}=1 / 2\left(Q_{i}+Q_{L}\right)-c(a)=Q_{L}+1 / 2 q(a) \Delta Q-c(a)
$$


The maximum payoff of the scientist is to maximize equation (1). Then, employer"s total payoff is $Q_{i}-1 / 2\left(Q_{i}+Q_{L}\right)=1 / 2\left(Q_{i^{-}} Q_{L}\right)$. Define equation (2) as employer"s expected payoff in SGN, conditional on scientist"s optimal action choices:

$$
D^{S G N}=1 / 2 E\left[Q_{i^{-}} Q_{L} \mid a=a^{S G N}\right]
$$

Then we have:

$$
S^{S G N}=Q_{L}+q\left(a^{S G N}\right) \Delta Q-c\left(a^{S G N}\right)
$$

3.1.2 SGI: In SGI, except a set wage $w$, employer can take the product away without giving the scientist any bonus. When expected this situation, scientist will refuse to take any high value actions, which makes $c(0)=0$. Then the employer will gain $Q_{L}$ and $\Delta Q=0$. So that the payoffs of the scientist and employer are:

$$
\begin{gathered}
U^{S G I}=w \\
D^{S G I}=Q_{L^{-}} w
\end{gathered}
$$

The total surplus in SGI will be:

$$
S^{S G I}=U^{S G I}+D^{S G I}=Q_{L}
$$

We note that SGI dominates SGN only when the net benefit from scientist"s actions in SGN is negative, $q\left(a^{S G N}\right) \Delta Q-c\left(a^{S G N}\right)<0$. This means that scientist"s choice of producing $Q_{H}$ leads to negative net benefit in SGN, so that both the scientist and employer have to choose SGI to produce $Q_{L}$. This case is possible in some institutional environment. We will further discuss this case in section 4 .

3.1.3 RGI: Unlike SGI, the relational employment contract in RGI may provide scientist incentives. So, an important part here is the payoff after reneging. We analyze trigger-strategy equilibrium, in which the party who did not renege refuses to make any new relational contract with the party who reneged. Because there are only two parties, this trigger-strategy assumption implies that these two people adopt SGN or SGI forever after one reneges. Here, the parties suspend the relational contract after reneging is allowed. So, when $S^{S G N}>S^{S G I}$, SGN will be adopted, while $S^{S G N}<S^{S G I}$, SGI will be adopted.

Let"s consider a relational compensation contract $\left(w, b_{H}, b_{L}\right) \equiv\left(w,\left\{b_{i}\right\}\right)$, where wage $w$ is paid by employer to scientist at the beginning of each period and bi is supposed to be paid when $Q=Q_{i}$, respectively (for $i=H, L$ ). Suppose the scientist believes that the employer will pay $b_{i}$ as promised (and that the scientist will not make any promised actions if $b_{i}<0$ ). If the scientist accepts the contract, he will choose $a^{R G I}$ to solve:

$$
U^{R G I}=w+q(a) b_{H^{\prime}}+(1-q(a)) b_{L^{-}} c(a)
$$

The expected employer payoff is:

$$
D^{R G I}=E\left[Q_{i}-w-b_{i} \mid a=a^{R G I}\right]=Q_{L}+q\left(a^{R G I}\right) \Delta Q-\left[U^{R G I}+c\left(a^{R G I}\right)\right]
$$

So the total surplus here is:

$$
S^{R G I}=U^{R G I}+D^{R G I}=Q_{L}+q\left(a^{R G I}\right) \Delta Q-c\left(a^{R G I}\right)
$$


RGI works if both parties honor the contract for all possible $Q_{i}$. The employer reneges if he refuses to pay the promised bonus $b_{i}$ to the scientist, just simply taking the product without paying anything. After reneging, when $S^{S G N}<S^{S G I}$, SGN will be adopted, then the employer will earn the payoff $D^{S G I}=Q_{L}$ in SGI, or while $S^{S G N}>S^{S G I}$, SGI will be adopted, then the employer earn the payoff $D^{S G N}$ in SGN.

Assume first that $S^{S G N}<S^{S G I}$, then if reneging occurs, both parties adopt SGI and the employer earns $D^{S G N}$. The employer will honor the relational contract as long as the present value of honoring the contract exceeds the present value of reneging. Since the present value of honoring the contract is $-b_{i}+1 / r D^{R G I}$, the employer will honor rather than renege on the relational contract when, for all $i$ :

$$
-b_{i}+1 / r D^{R G I} \geqslant 1 / r D^{S G I}
$$

The scientist reneges on the relational contract by refusing to accept a promised payment bi when it was offered (or by refusing to make a promised actions if $b_{i}<0$ ), just earning $\mathrm{U}^{\mathrm{RGI}}$. So that the scientist will honor rather than renege on the relational contract when, for all $i$ :

$$
b_{i}+1 / r U^{R G I} \geqslant 1 / r U^{S G I}
$$

Combining (10) and (11) yields a necessary condition for RGI works when $S^{S G N}<S^{S G I}$ :

$$
\max b_{i}-\min b_{i} \leqslant 1 / r\left(S^{R G I}-S^{S G I}\right)
$$

$S^{S G N}>S^{S G I}$ is assumed next, so that if reneging occurs, then SGN will be adopted, the scientist and employ will respectively earn $U^{S G N}$ and $D^{S G N}$. Then the right-hand side of (10) becomes $1 / r D^{S G N}$, and the right-hand side of (11) becomes $1 / r U^{S G N}$, so combining the extreme versions of the resulting incentive constraints yields a necessary condition for RGI works when $S^{S G N}>S^{S G I}$ :

$$
\max b_{i}-\min b_{i} \leqslant 1 / r\left(S^{R G I}-S^{S G N}\right)
$$

Combining (12) and (13) yields a necessary and sufficient condition for RGI works:

$$
\max b_{i}-\min b_{i} \leqslant 1 / r\left(S^{R G I}-\max \left(S^{S G I}, S^{S G N}\right)\right)
$$

For any $\max b_{i}$ and $\min b_{i}$, a fixed wage $w$ can always be chosen that satisfies (10) and (11) (when $S^{S G N}>S^{S G I}$ ) or their analogs (when $S^{S G N}<S^{S G I}$ ). The left-hand side of (14) is the maximum total temptation to renege on the relational contract. The right-hand side of (14) is the present value of the net total surplus. With the feasibility constraint (14), the efficient relational contract maximizes the total surplus in (9).

3.1.4 RGN: In RGN. If the promised $b_{i}$ is greater than the price that would be negotiated in SGN, $1 / 2\left(Q_{i}+Q_{L}\right)$, The employer would be better off this period to reneged on the relational contract. Similarly, if the promised bonus bi is less than $1 / 2\left(Q_{i}+Q_{L}\right)$, then the scientist would be better off this period to reneged. Thus, the key difference between RGN and RGI is that the product"s value in its low value, $Q_{L}$, affects the reneging decision in RGN.

If the scientist believes that the employer will honor the relational contract, then the scientist will choose $a^{R G N}$ to solve $U^{R G N}=w+q(a) b_{H}+(1-q(a)) b_{L}-c(a)$, The expected employer"s payoff is then $D^{R G N}=E\left[Q_{i}-s-b_{i} \mid a=a^{R G N}\right]$, so the total surplus in RGN is: 


$$
S^{R G N}=U^{R G N}+D^{R G N}=Q_{L}+q(a) \Delta Q-c\left(a^{R G N}\right)
$$

Note that a given relational contract $\left(w,\left\{b_{i}\right\}\right)$ produces the same actions and the same surplus in RGN as in RGI. However, we will show that the temptation to renege in RGN differs from RGI.

Once $Q_{i}$ has been realized, the employer is supposed to receive the net payoff $Q_{i}-b_{i}$. If he reneges in RGN, he negotiates with the scientist to buy the product for SGN price of $1 / 2\left(Q_{i}+Q_{L}\right)$ instead of for $b_{i}$, realizing a current payoff of $Q_{i}-1 / 2\left(Q_{i}+Q_{L}\right)$. Similarly, once $Q_{i}$ has been realized, the scientist is supposed to sell the product for the price $b_{i}$. If he reneges, he negotiates to sell the product for the price of $1 / 2\left(Q_{i}+Q_{L}\right)$.

When $S^{S G N}>S^{S G I}$, no matter who has reneged, both will choose SGN. Then, the employer will honor rather than renege in RGN when, for all $i$ :

$$
Q_{i}-b_{i}+1 / r D^{R G N} \geqslant 1 / 2\left(Q_{H^{-}} Q_{L}\right)+1 / r D^{S G N}
$$

We rewrite it as:

$$
b_{i}-1 / 2 Q_{i} \leqslant 1 / r\left(D^{R G N}-D^{S G N}\right)
$$

Similarly, the scientist will honor rather than renege in RGN when, for all $i$ :

$$
b_{i}-1 / 2 Q_{i} \geqslant 1 / r\left(U^{R G N}-U^{S G N}\right)
$$

Combining (16) and (17) yields a single necessary condition for RGN works when:

$$
\max \left(b_{i}-1 / 2 Q_{i}\right)-\min \left(b_{i}-1 / 2 Q_{i}\right) \leqslant 1 / r\left(S^{R G N}-S^{S G N}\right)
$$

When $S^{S G N}<S^{S G I}$, then no matter who has reneged, both parties will choose SGI. In this case, a single necessary condition to make RGN work becomes:

$$
\max \left(b_{i}-1 / 2 Q_{i}\right)-\min \left(b_{i}-1 / 2 Q_{i}\right) \leqslant 1 / r\left(S^{R G N}-S^{S G I}\right)
$$

Combining the extreme versions of the resulting incentive constraints yields a necessary as well as sufficient condition to make RGN work:

$$
\max \left(b_{i}-1 / 2 Q_{i}\right)-\min \left(b_{i}-1 / 2 Q_{i}\right) \leqslant 1 / r\left(S^{R G N}-\left\{S^{S G N}, S^{S G I}\right\}\right)
$$

The left-hand side of (20) is the maximum total temptation to renege in RGN, and the right-hand side is the present value of the total surplus from continuity RGN. With the feasibility constraint (20), the total surplus in (15) can be maximized.

With the basic models above, given functional forms for $q(a)$ and values for $\Delta Q$ and $r$, we can determine which governance structures will dominate.

\subsection{Comparative Analysis of the Four Governance Structure Forms in a Special Case}

Here, we use a special case of the basic models developed in sub-section 3.1 to establish our main propositions. Assume that a has two components, $q(a)$ is linear, and $c(a)$ is quadratic:

$$
\begin{aligned}
& a=\left(a_{1}, a_{2}\right) \\
& q(a)=q_{1} a_{1}+q_{2} a_{2}
\end{aligned}
$$




$$
c(a)=1 / 2 a_{1}^{2}+1 / 2 a_{2}^{2}
$$

Where, $q_{1} \geqslant 0, q_{2} \geqslant 0$ and $q_{1} \neq q_{2}$.

For this case, the first-best (FB) actions are:

$$
\begin{aligned}
& a_{1}^{F B}=q_{1} \Delta Q \\
& a_{2}^{F B}=q 2 \Delta Q
\end{aligned}
$$

In SGN, the scientist chooses $a_{1}$ and $a_{2}$ to maximize $Q_{L}+1 / 2 q(a) \Delta Q-c(a)$, which yields:

$$
\begin{gathered}
\mathrm{a}_{1}{ }^{\mathrm{SGN}}=\mathrm{q}_{1} \Delta \mathrm{Q} \\
\mathrm{a}_{2}{ }^{\mathrm{SGN}}=\mathrm{q}_{2} \Delta \mathrm{Q}
\end{gathered}
$$

In SGI, the scientist has no incentive to choose high price actions, thus, $a_{1}{ }^{S G I}=a_{2}{ }^{S G I}=0$.

When relational contract is chosen, the bonus differs according to the quality of the products, which means, if $Q=Q_{H}$, employer will pay the scientist at a price of $\Delta b=b_{H^{-}} b_{L}$. So that when $Q$ gets to the maximum value $Q_{H}$, no further bonus will be paid. With the relational contract, the scientist will choose $a_{1}$ and $a_{2}$ to maximize $\left(w+b_{L}\right)+q(a) \Delta b-c(a)$, and we yield:

$$
\begin{aligned}
& \mathrm{a}_{1}{ }^{\mathrm{RG}}=\mathrm{q}_{1} \Delta \mathrm{b} \\
& \mathrm{a}_{2}{ }^{\mathrm{RG}}=\mathrm{q}_{2} \Delta \mathrm{b}
\end{aligned}
$$

Here " $R$ " refers to relational contract and "G" refers to asset generality, as we have shown before, the total surplus of RGN and RGI is equal.

Equation (14) has provided the necessary and sufficient conditions for RGI to be efficient, with $b_{i}(i=H, L)$, the following two situations need to be taken into account:

1. When $b_{H}>b_{L}, \max \left(b_{i}\right)=b_{H}, \min \left(b_{i}\right)=b_{L}$, the reneging temptation is $\Delta b$, in which $\Delta b=b_{H^{-}} b_{L}>0$;

2. When $b_{L}>b_{H}, \max \left(b_{i}\right)=b_{L}, \min \left(b_{i}\right)=b_{H}$, the reneging temptation is $-\Delta b$, in which $\Delta b=b_{H^{-}} b_{L}<0$

The two situations can be writen as $|\Delta b|$, then we yield:

$$
|\Delta b| \leqslant 1 / r\left(S^{R G I}-\max \left(S^{S G I}, S^{S G N}\right)\right)
$$

Equation (20) has provided the necessary and sufficient conditions for RGN to be efficient, we note:

(a) $b_{H^{-}}-1 / 2 Q_{H}=b_{H^{-}}-1 / 2 Q_{L^{-}} 1 / 2 \Delta Q=(\Delta b-1 / 2 \Delta Q)+b_{L^{-}} 1 / 2 Q_{L}$

(b) $b_{L^{-}} 1 / 2 Q_{L}$

With $b_{i}(i=H, L)$, we need to take the following two situations into account:

1. When $\Delta b>1 / 2 \Delta Q$, then, $\max \left(b_{i^{-}}-1 / 2 Q_{i}\right)=b_{H^{-}} 1 / 2 Q_{H}, \min \left(b_{i^{-}}-1 / 2 Q_{i}\right)=b_{L^{-}} 1 / 2 Q_{L}$, and the reneging temptation will be $\Delta b-1 / 2 \Delta Q$;

2. When $\Delta b<1 / 2 \Delta Q$, then, $\max \left(b_{i^{-}}-1 / 2 Q_{i}\right)=b_{L^{-}} 1 / 2 Q_{H}, \min \left(b_{i^{-}}-1 / 2 Q_{i}\right)=b_{H^{-}} 1 / 2 Q_{L}$, and the reneging temptation will be $-(\Delta b-1 / 2 \Delta Q)$

The two situations can be writen as $|\Delta b-1 / 2 \Delta Q|$, then we yield: 
With (22), we can achieve the first-best in a relational contract if and only if $\Delta b=\Delta Q$, so (14') implies RGI can achieve a maximum total surplus if and only if:

$$
\Delta Q \leqslant 1 / r\left(S^{R G I}-\max \left(S^{S G I}, S^{S G N}\right)\right)
$$

Similarly, (20`) implies RGN can achieve a maximum total surplus if and only if:

$$
1 / 2 \Delta Q \leqslant 1 / r\left(S^{R G N}-\max \left\{S^{S G N}, S^{S G I}\right\}\right)
$$

Based on this special case, we can prove the following propositions:

Proposition 1. Comparing to the other three forms of governance structures, when the asset nature is relational generality, SGN dominates in the achieving to maximum total surplus. Formally, given $q_{1}$ and $q_{2}$, if the asset nature is relational generality, such that if $\Delta Q>0$, then $\mathrm{SGN}$ is the dominated form.

Proof. Given Lemmas 1 and 3 below.

Lemma 1. when the asset nature is relational generality, the governance structure form "non-integration + non-relational contract" always dominates the form "integration + nonrelational contract". That is, given $q_{1}$ and $q_{2}$, such that if $\Delta Q>0$, then $S^{S G N}>S^{S G I}$.

Proof. Because $\Delta Q>0$, then $a_{1}^{S G N}=q_{1} \Delta Q>0, \quad a_{2}^{S G N}=q_{2} \Delta Q>0, \quad$ so that $\max$ $S^{S G N}=Q_{L}+1 / 8 \Delta Q\left(q_{1}{ }^{2}+q_{2}{ }^{2}\right)$; as to SGI, for $a_{1}^{S G I}=a_{2}{ }^{S G I}=0$, and $S^{S G I}=Q_{L}$. Then $S^{S G N}$ $S^{S G I}=1 / 8 \Delta Q\left(q_{1}{ }^{2}+q_{2}{ }^{2}\right)>0$, so $S^{S G N}>S^{S G I}$.

Lemma 2. when the asset nature is relational generality, the governance structure form "non-integration + relational contract" always dominates the form "integration + relational contract". That is, given $q_{1}$ and $q_{2}$, such that if $\Delta Q>0$, although the total surplus of RGN and RGI are equal, $S^{R G N}=S^{R G I}$, the reneging temptation of former case is less than latter case, which makes RGN dominate RGI.

Proof. From (14') and (20') we can see that, with $\Delta Q>0$, then whatever the value of the interest $r$ is, the reneging temptation $|\Delta b-1 / 2 \Delta Q|$ of RGN is always less than the reneging temptation $|\Delta b|$ of RGI.

Lemma 3. when the asset nature is relational generality, the governance structure form "non-integration + non-relational contract" always dominates the form "non-integration + relational contract". That is, given $q_{1}$ and $q_{2}$, such that if $\Delta Q>0$, SGN and RGN have the same total surplus, but SGN has no reneging temptation, while RGN always has reneging temptation.

Proof. We can learn from the beginning of this section that, $a_{1}{ }^{F B}=a_{1}^{S G N}=q_{1} \Delta Q$, $a_{2}^{F B}=a_{2}{ }^{S G N}=q_{2} \Delta Q$, when relational contract exists and given (22), such that if $\Delta b=\Delta Q$, then the maximum total surplus can be yielded, so that $a_{1}{ }^{R G}=a_{1}{ }^{F B}=a_{l}{ }^{S G N}=q_{1} \Delta Q$, $a_{2}{ }^{R G}=a_{2}{ }^{F B}=a_{2}{ }^{S G N}=q_{2} \Delta Q$, and $S^{S G N}=S^{R G N}$. From lemma 1 we can learn that $S^{S G N}$ is always larger than $S^{S G I}$, thus as (24), $S^{R G N}-\max \left\{S^{S G N}, S^{S G I}\right\}=S^{R G N}-S^{S G N}=0<1 / 2 \Delta Q$, then the necessary and sufficient conditions of not reneging in RGN cannot exist.

Proposition 1 is proofed.

Proposition 2. When the asset nature is relational generality, with opportunism, the governance structure form "integration + relational contract" cannot exist. That is, given 
$q_{1}$ and $q_{2}$, such that if $\Delta Q>0$, then given any $r>0$, no $\Delta b$ can satisfy the not reneging condition (14) for RGI.

Proof. To achieve the maximum total surplus, $S^{S G N}$ is always larger than $S^{S G I}$, thus $\max \left\{S^{S G N}, S^{S G I}\right\}$ is always equal to $S^{S G N}$. Then (14) can be rewritten as:

$$
|\Delta b| \leqslant 1 / r\left(S^{R G I}-S^{S G N}\right)
$$

To realize the first-best, the total surplus of SGN is always equal to RGI"s, then on the right-hand side of (14“), the maximum value of $S^{\mathrm{RGI}}-S^{\mathrm{SGN}}$ is zero, that is, given any $r>0$, $S^{R G I}-S^{S G N} \leqslant 0$. While on the left-hand side of $\left(14^{\prime \prime}\right)$, unless $\Delta b=0$, or else the existence of $(14$ “) is impossible.

However, when $\Delta b=0$, then whatever the scientist produces $Q_{H}$ or $Q_{L}$, the employer will always give a fixed incentive w. In this case, the scientist"s best choice is not taking any high price action but produce $Q_{L}$, which provides a smaller total surplus than $S^{S G N}$, and makes $S^{R G I}-S^{S G N}<0$. For this moment, the left-hand side of (14") is bigger than zero, while the right-hand side is smaller than zero, this inequation fails to exist.

Proposition 2 is proofed.

Proposition 3. When the asset nature is relational generality, with opportunism, the governance structure form "non-integration + relational contract" cannot exist. That is, given $q_{1}$ and $q_{2}$, such that if $\Delta Q>0$, then given any $r>0$, no $\Delta b$ can satisfy the not reneging condition (20') for RGN.

Proof. To achieve the maximum total surplus, $S^{S G N}$ is always larger than $S^{S G I}$, thus $\max \left\{S^{S G N}, S^{S G I}\right\}$ is always equal to $S^{S G N}$. Then (20') can be rewritten as:

$$
|\Delta b-1 / 2 \Delta Q| \leqslant 1 / r\left(S^{R G N}-S^{S G N}\right)
$$

To realize the first-best, the total surplus of SGN is always equal to RGN"s, then on the right-hand side of (20"), the maximum value of $S^{R G N}-S^{S G N}$ is zero, that is, given any $r>0$, $S^{R G N}-S^{S G N} \leqslant 0$. While on the left-hand side of $\left(20^{\prime \prime}\right)$, unless $\Delta b=1 / 2 \Delta Q$, or else the existence of (14") is impossible.

However, when $\Delta b=1 / 2 \Delta Q, S^{R G N}$ cannot achieve its maximum value, and makes $S^{R G N}$ $S^{S G N}<0$. In this case, the left-hand side of $\left(20^{\prime \prime}\right)$ is equal to zero, while the right-hand side is smaller than zero, this inequation fails to exist.

Proposition 3 is proofed.

\section{Discussion}

First, the two governance structure forms SGN and SGI can be realized without incentives to renege, while the other two forms "RGN" and "RGI" cannot be realized without incentives to renege, for given a relational contract " $\mathrm{R}$ ", no $\Delta b$ satisfies the constrains for the two parties in RGN or RGI not to renege. As a result, it had better not adopt any relational contract in the governance structure of little science.

Second, although SGN and SGI can be realized without reneging, there is a fundamental difference between them. SGN is constructed on the asset nature, in which the highest value $Q_{H}$ of the knowledge product and a maximum total surplus can be yielded. Thus, the best governance structure of little science is "SGN". SGI doesn"t construct on the basis of asset nature. The reason that SGI can be realized without reneging is that " $\mathrm{S}$ " can avoid the existence of reneging temptation. In SGI, whatever $Q_{i}$ is, the payoff is w only. However, within the governance structure "I", the scientist has to 
follow the orders given by the employer. So that the scientist, mundane and opportunism, will choose to produce $Q_{L}$ as dominated actions in SGI.

At last, although it is irrelevant to the asset nature, it doesn"t imply that SGI will not be adopted. By analyzing SGI, we have established contact with the observations of institutionalism. In the studies of institutionalism, the formal institution or formal organization is considered to gain legitimacy in a given social environment, but not for the actual organizational operation. In a given social environment, when "I" has to be adopted as a demand for legitimacy, then the existence of "I" is a must. In this premise, both parties can only adjust the informal organizational structure. When both parties choose relational contract "R", as we show before, the RGI form cannot be sustained. Once reneged, the only fallback is SGI. In this case, we may observe that reneging happens from time to time or lots of low value products will be produced.

\section{The Policy Implications for the Little Science in China}

Many socialists have put forward insights on the governance logic of Chinese administrative system. Zhou points out that, during the period of Great Leap Forward Movement, the administrative system of China was like a "Tournament System", in this system, the central government had taken most of the resource in control. If the local governments wanted to obtain these resources, they had to finish the tasks assigned by the central government [17]. Qu states that China has formed a state governance mode named "Project System", in this mode, the projects guidelines made by different government departments have become "race standards" of tournament in the new period. With these race standards, departments of lower level compete with each other to win the projects and resources, while the resources and the rules of the games are possessed by the superior departments [18].

We can observe that most of the science research institutions are public institutions with administrative ranks, including the universities which representative of little science. So that most of the science institutions are parts of the whole Chinese administrative system, if these institutions want to obtain resources they need, they have to follow the state governance mode of this system to gain legitimacy first.

Take China's universities as example, if a university wants to obtain the resources for development, the numbers of the achievements in science are one of the main factors that help this university win the projects and funds. In these projects tournament, the university as an employer will assign the numbers as tasks to scientists and the payoff of the scientists are linked with these numbers. The more numbers can be finished, the more bonus the scientist will get upon a basic wage, which is a relational contract " $R\left(w,\left\{b_{i}\right\}\right)$ ". Though the university as an employer doesn't stipulate the subjects for the scientist, he actually intervenes scientist's choice making and uses the scientist's product. Obviously, the formal governance structure in this university is "I". Then, as a representative institution of little science, the governance structures of China's universities are "RGI". Due to "I" cannot be adjusted in the Chinese institution environment for now, when reneging happens in RGI in little science, "SGI" is the only fallback. As we have shown in the Discussion in section 4, if the governance structure of little science is built on RGI and its only fallback is SGI, then high value product will hardly be produced. This is the reason why Little Science doesn't meet the development demand of modern science in China. To rebuild the governance structure, de-administration of little science is needed.

\section{Acknowledgements}

This work was supported by Harbin Institute of Technology's "Big science facilities special pre-research project" (AUEA5760000511). 


\section{References}

[1] D.N.Shen, "The comments about controversy over Big Science and Little Science", Science Technology and Dialectics (2009), Vol. 26, No. 1, pp. 101-112.

[2] S.H. Zhong, "On whole-nation system for science and technology", Studies in Science of Science (2009), Vol. 27, No. 12, pp.1785-1791.

[3] D.Price, Little Science Big Science, Columbia University, New York (1963).

[4] O.E. Williamson, "Comparative economic organization: the analysis of discrete structural alternatives", Administrative Science Quarterly (1991), Vol. 36, No. 2, pp. 269-296.

[5] M.H. Riordan and O.E. Williamson, "Asset specificity and economic organization", International Journal of Industrial Organization (1985), Vol. 4, No. 3, pp. 365-378.

[6] B. Klein, R.G. Crauford and A.A. Alchian, "Vertical Integration, Appropriable Rents, and the Competitive Contracting Process", Journal of Law and Economics (1978), Vol. 21, No. 2, pp. 297-326.

[7] J.W. Meyer and B. Rowan, "Institutionalized Organizations: Formal Structure as Myth and Ceremony", American Journal of Sociology (1977), Vol. 83, No. 2, pp. 340-363.

[8] I. R. Macneil, "Relational Contract: What we do and do not know", Wis. L. Rev. (1985), Vol.8, No. 3, pp. 483-525.

[9] G. Baker, R. Gibbons and K.J. Murphy, "Relational contracts and the theory of the firm", The Quarterly Journal of Economics (2002), Vol.177, No. 1, pp. 39-84.

[10] Y. Lee, S.T. Cavusgil, "Enhancing alliance performance: The effects of contractual-based versus relational-based governance", Journal of Business Research (2006), Vol.59, No. 8, pp. 896-905.

[11] L. Poppo, K. Z. Zhou and T. R. Zenger, "Examining the Conditional Limits of Relational Governance: Specialized Assets, Performance Ambiguity, and Long-Standing Ties", Journal of Management Studies (2008), Vol. 45, No.7, pp.1195-1216.

[12] L. Poppo and T. Zenger, "Do formal contracts and relational governance function as substitutes or complements?", Strategic Management Journal (2002), Vol. 23, No. 8, pp. 707-725.

[13] G. Wang, "Invisible hand and rules in science: from perspective economics of science", Studies in Science of Science (2007), Vol. 25, No. 5, pp. 836-841.

[14] H.F. Fang and J.L. Chen, "On the revealing of the priority theory to the human resources management probing into the Merton"s priority theory in sociology of science", Studies in Science of Science (2005), Vol. 23, No. 12, pp. 29-34.

[15] O. E. Williamson, "The theory of the firm as governance structure: from choice to contract", Journal of Economic Perspectives (2002), Vol. 16, No. 3, pp. 171-195.

[16] G.L. Jia and H.F. Liu, "The rise of economics of science and the latest development", Foreign Social Science (2003), No. 2, pp. 32-34.

[17] F.Z. Zhou, "The Tournament System", Sociological Studies (2009), No.3, pp. 54-77.

[18] J.D. Qu, "Project System: A new system of state governance", Social Science in China (2012), No. 5, pp. 113-130. 
International Journal of Security and Its Applications

Vol.9, No.10 (2015) 